\title{
Enlargement of the narrow aortic root and oblique insertion of a St Jude prosthesis
}

\author{
ROBIN H KINSLEY, MANUEL J ANTUNES, JOSEPH K MCKIBBIN \\ From the Departments of Cardiothoracic Surgery and Medicine, University of the Witwatersrand, Fohannesburg, \\ South Africa
}

SUMmary The aortic root was enlarged and a St Jude Medical valve obliquely inserted in 11 patients. Prosthetic valve sizes at least one, but often two, sizes larger than that which would normally be accommodated by the dimensions of the host aortic annulus were used. Closure of the valve was not affected by obliquity. This simple technique is recommended whenever the host aortic annulus does not allow the insertion of a substitute valve of an appropriate size.

Modern mechanical prostheses of the tilting disc type have largely eliminated the haemodynamic inadequacies of the earlier substitute valves which had a central occluder. ${ }^{1}$ The St Jude Medical valve, with a bileaflet design, has excellent haemodynamic characteristics even in the smaller sizes. ${ }^{2}$ Nevertheless, the narrow aortic root remains an obstacle to the satisfactory functional correction of aortic valve disease. One of us (RHK) described a technique for inserting a prosthesis larger than that which would normally be accommodated by the dimension of the host aortic annulus. ${ }^{3}$ The technique comprised two essential components: firstly, widening of the aortic annulus, including the subannular and supra-annular regions and, secondly, oblique insertion of the prosthesis.

We report our experience with enlargement of the aortic root and oblique implantation of the St Jude prosthesis.

\section{Method}

After institution of cardiopulmonary bypass and aortic cross-clamping a straight aortotomy was performed towards the non-coronary sinus. The aortic valve was excised. When the size of the aortic annulus precluded the insertion of a $23 \mathrm{~mm}(21 \mathrm{~mm}$ for small patients) prosthesis the aortotomy was prolonged across the aortic annulus in the region of the noncoronary sinus into the subaoric "curtain". Occasionally, the anterior leaflet of the mitral valve was incised. The roof of the left atrium is a close relation to the aortic annulus and left atrium may be entered

Accepted for publication 21 June 1983 with the incision. Figure of eight sutures were passed through the original aortic annulus and then through about two thirds of the circumference of the prosthesis. The defect created in the left ventricular outflow tract, aortic annulus, and aorta was widened with a triangular or teardrop shaped patch of pericardium, expanded polytetrafluoroethylene, ${ }^{\star}$ or dacron. A 4/0 prolene suture was used, beginning at the lower end of the aortotomy and its subannular extension, and continued upwards on both sides of the incision until about $10 \mathrm{~mm}$ above the level of the annulus. Any opening in the left atrium or the mitral valve or both was closed by incorporation in the suture line. The prosthesis was tied down using the suture previously placed in the host aortic annulus. The remaining one third of the prosthetic ring was then sutured to the patch with a series of multiple horizontal mattress sutures reinforced with a single 2 to $3 \mathrm{~cm}$ long teflon felt pledget on the outside of the patch. The prosthesis lay in an oblique plane about 5 to $10 \mathrm{~mm}$ above the level of the host aortic annulus in the region of the non-coronary sinus (Fig. 1). The degree of obliquity of the valve may be modified to accommodate different prosthetic valve sizes. Closure of the aortotomy was completed with suture of the upper half of the patch.

\section{Patients}

From January 1980 to July 1982 , aortic root enlargement and oblique insertion of the St Jude Medical valve was used in 11 patients (mean age

*Gore-Tex, registered trademark of W L Gore and Associates, Inc, Eklton, Maryland, USA. 


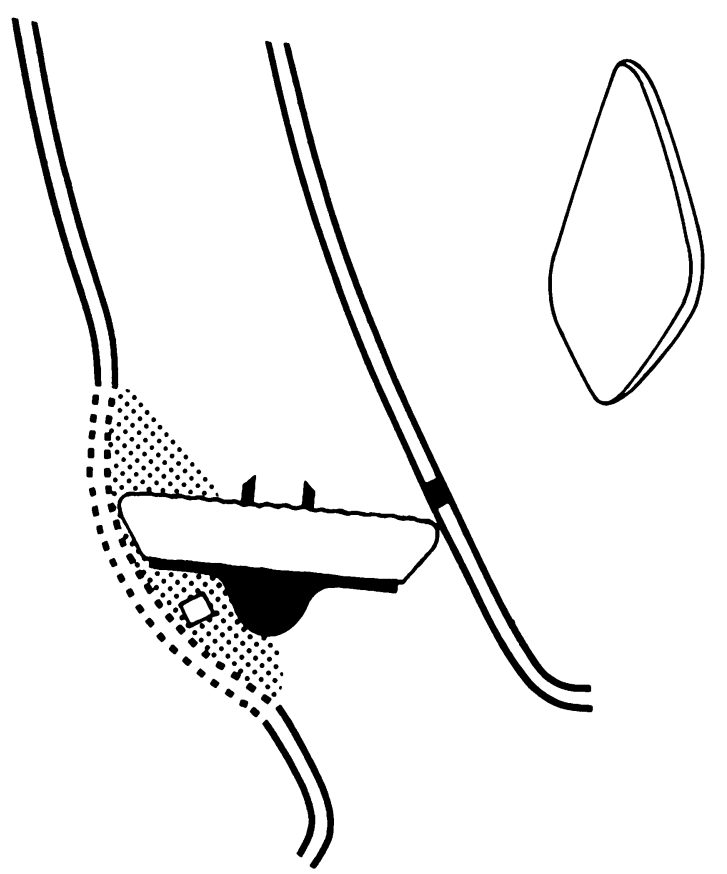

Fig. 1 Technique of aortic root enlargement and oblique insertion of the St Fude Medical prosthesis.

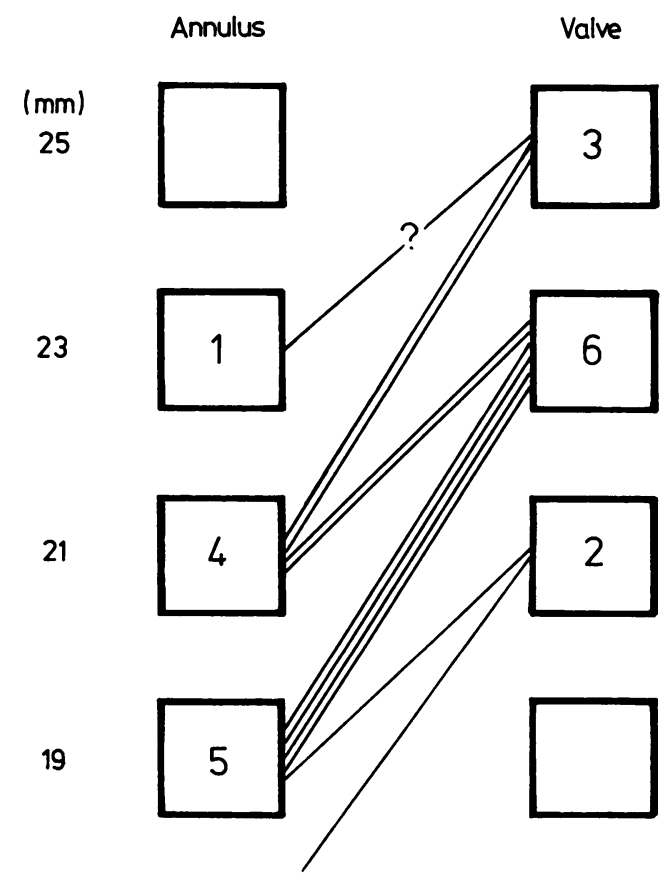

Fig. 2 Size of prostheses implanted compared with the dimensions of the intact host aortic annulus.
$51(\mathrm{SD} \pm 24)$ years $)$. In three patients the annulus was congenitally narrowed, and in two there was rheumatic aortic stenosis. Five patients had had previous aortic valve surgery, three with prosthetic valve insertion. In one patient an excessively large valve $(25 \mathrm{~mm})$ was inadvertently chosen for an annulus which measured only $23 \mathrm{~mm}$.

Supra-annular widening of the aortic root alone with oblique insertion of the prosthesis was carried out in two patients. The remaining nine patients had aortic and annular enlargement. An autologous pericardial patch was used in seven patients and polytetrafluoroethylene in two; bovine pericardium $†$ and dacron (one case each) completed the series. In all patients a prosthesis at least one size larger (in seven, two sizes larger) than that which would normally be accommodated by the dimensions of the intact host aortic annulus was implanted (Fig. 2).

Intraoperative pressures were measured before and after valve replacement. The mean peak systolic aortic transvalvular gradient decreased from $47 \mathrm{~mm} \mathrm{Hg}$ (range 5 to 90) to $3 \mathrm{~mm} \mathrm{Hg}$ (range 1 to 15). Left atrial pressure tracings did not indicate any adverse effect of the incision into the anterior leaflet on mitral valve function. Postoperatively this was confirmed clinically.

One patient undergoing triple valve replacement died of multiple organ failure nine days after surgery. The remaining patients survived the operation and were followed for seven to 37 (mean 22) months. All were alive and well at this time. There were no thromboembolic episodes or perivalvular leaks. All patients were examined clinically and in none was valve dysfunction diagnosed. Cinefluoroscopy was performed in all patients. Full and equal excursion of both valve leaflets was evident in all patients (Fig. 3).

\section{Discussion}

The technique, as originally described, ${ }^{3}$ has been used in our unit with three different prostheses: the Björk-Shiley, Hall-Kaster, and St Jude Medical †Shiley Laboratories, Anaheim, California.

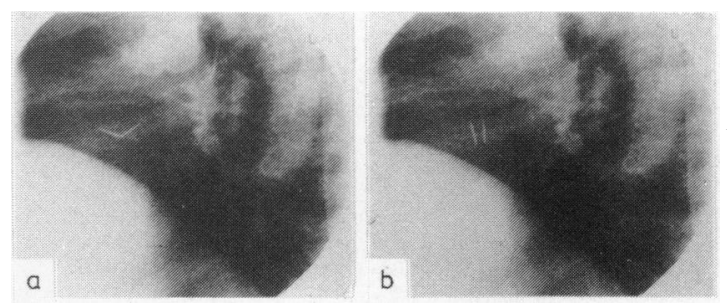

Fig. 3 Cineftuoroscopy showing valve (a) closed and (b) fully open. Full and equal excursion of both leaflets was evident in all patients. 
valves. Our total experience is of more than 60 cases. It has been particularly useful in two groups of patients: (a) in children in whom it is desirable to implant an adult sized prosthesis to allow for growth, and (b) in adults with a narrow aortic root.

The implantation of a larger prosthesis is made possible by widening the aortic root and obliquely inserting the valve in relation to the plane of the host aortic annulus. No complications were encountered with the Björk-Shiley prosthesis. With the HallKaster valve, however ( $75 \%$ open position disc angle), oblique insertion of the prosthesis occasionally resulted in the occluder opening beyond the axis of flow. ${ }^{4}$ Closure of the valve is compromised with resultant free aortic regurgitation, which may be intermittent. Although not encountered, this complication remains a theoretical possibility with the Björk-Shiley prosthesis.

One of the uncertainties of the technique has been the fate of the pericardial patch. ${ }^{5}$ We have since reoperated on five patients who underwent enlargement of the aortic root with an autologous pericardial patch. Reoperation was performed two to six years after initial valve replacement for removal of a thrombosed Björk-Shiley prosthesis or mitral valve replacement. There were no instances of patch aneurysm, but in one patient the patch was mildly calcified. Apart from the two suture lines the pericardium was usually almost indistinguishable from the true aortic wall.

Similar techniques have been described elsewhere. ${ }^{6}$ In our method, however, the obliquity of the valve allows the insertion of a prosthesis larger than that which would be possible as a result of simple widening of the aortic annulus. Moreover, in vitro studies, ${ }^{7}$ and our in vivo observations, have shown that obliquity does not affect the proper closure and func- tioning of the St Jude Medical valve. We recommend this simple reproducible technique in all patients in whom the dimension of the aortic annulus does not allow insertion of an appropriately sized prosthesis. Indeed, we now tailor the size of the prosthesis inserted to the expected stroke volume of the patient.

\section{References}

1 Björk VO, Henze A. Ten years' experience with the Björk-Shiley tilting disc valve. $\mathcal{F}$ Thorac Cardiovasc Surg 1979; 78: 331-42.

2 Emery RW, Mettler E, Nicoloff DM. A new cardiac prosthesis: the St Jude Medical cardiac valve. Circulation 1979; 60 (suppl I): 48-54.

3 Kinsley RH. The narrow aortic annulus. A technique for inserting a larger prosthesis. Am Heart f 1977; 93: 759 61.

4 Antunes MJ, Colsen PR, Kinsley RH. Intermittent $\overrightarrow{-}$ aortic regurgitation following aortic valve replacement with the Hall-Kaster prosthesis. $\mathcal{F}$ Thorac Cardiovasc Surg 1982; 84: 751-4.

5 Stenseth JH, Danielson GK, McGoon DC. Pericardial patch enlargement of the aortic outflow tract. $\mathcal{F}$ Thorac Cardiovasc Surg 1971; 62: 442-8.

6 Manouguian S, Seybold-Epting W. Patch enlargement of the aortic valve ring by extending the aortic incision into the anterior mitral leaflet: a new operative technique. $\mathcal{F}$ Thorac Cardiovasc Surg 1979; 78: 402-12.

7 Kasagi Y, Wada J. Hemodynamic characteristics of the St Jude Medical valve in mock circulation. In: Proceedings of the Second International Symposium on the St fude Medical Prosthesis. 1981: 245-64.

Requests for reprints to Professor R H Kinsley, Department of Cardio-Thoracic Surgery, University of the Witwatersrand Medical School, York Road, Parktown 2193, South Africa. 